\title{
Conductivity Percolation of Carbon Nanotubes in Polyacrylamide Gels
}

\author{
Önder Pekcan and Gülşen Akın Evingür \\ Kadir Has University, \\ İstanbul Technical University, \\ Turkey
}

\section{Introduction}

Polymer composites can be used in many different forms in various areas ranging from structural units in the construction industry to the composites of the aerospace applications. The extraordinary properties of carbon nanotubes make them very promising and favorable as fillers for fabrication of a new class of polymeric heterostructures. Polymer matrices have been widely exploited as a medium for carbonnanotubes (CNTs) which have been described as effective conducting fillers for polymers. Nanotubes can be described as long and slender fullerenes, in which the walls of the tubes are hexagonal carbon (graphite structure). There are two main types of carbon nanotubes (CNT) known as single (SWCNT) and multiwalled (MWCNT). Since the discovery of carbon nanotubes (CNT) in 1991 by lijima (lijima, 1991), single- wall carbon nanotubes (SWNT) and multiwalled carbon nanotubes (MWNT) have attracted great interests throughout the academic and industrial applications. Therefore, many researchers are focused on the development of CNT-based polymer materials that utilize the carbon nanotubes characteristics and properties, such as the high strength and stiffness of the CNTs are used for developing superior polymer composites for structural applications which are lighter, stronger, and tougher than any polymer-based material. There are several important requirements for an effective improvement of CNT-based composites' properties, such as: a large aspect ratio of a filler, good exfoliation and dispersion of nanotubes, and good nanotube-nanotube and nanotube-polymer interfacial bonding. Numerous studies have shown already, that an effective performance of the carbon nanotubes in composites for a variety of applications strongly depends on the ability to disperse the CNTs homogenously throughout the matrix. Good interfacial bonding and interactions between nanotubes and polymers are also necessary conditions for improving mechanical properties of the composites. Because of their high mechanical strength, high aspect ratio, small diameter, light weight, high electrical and thermal conductivities, and high thermal and air stabilities, CNT have been found many applications in the industrial world (Chang, 2006; Hu, 2006). Polymer composites with carbon nanotube addition are one of the research subjects which attract huge attention in recent years (Lau, 2002\& Thostenson, 2001). Thermoplastic polymers are usually used as an insulating material because of their low electrical conductivity properties $\left(\approx 10^{-15} \mathrm{~S} / \mathrm{m}\right)$. Dispersing the conducting filler such as carbon black and CNT in polymer phase forms conductive polymer composites. Electrical 
resistivities of polymer-MWCNT composites which are strongly dependent on the volume or mass fractions of the CNTs may vary between $10^{16}$ to several ohms. At low volume or mass fractions, the resistivity remains very close to the resistivity of pure polymer. Insulating polymers are transformed to conductive composites by addition of CNTs above a critical concentration threshold (known as percolation threshold). When the positions of CNTs in the polymer matrix form a conducting network, the conductivity of composite sharply increases. This phenomenon is known as percolation and can be well explained by percolation theory (Stauffer, 1994). Percolation threshold can be determined by measuring the resistivity variations in composites. Electrical percolation thresholds for some MWCNT and SWCNT polymer-composites were reported as ranging from 0.0021 to $15 \mathrm{wt} \%$ (Bauhofer, 2009). Studies on polymer-CNT composites show that their electrical, mechanical and thermal properties are improved by addition of CNTs (Chang, 2006; Choi, 2007; Du, 2004; Gao, 2007; Park, 2007). The first polymer nanocomposites using carbon nanotubes as filler were reported in 1994 by Ajayan (Ajayan, 1994). Earlier nanocomposites were used nanoscale fillers such as carbon blacks, silicas, clays, and carbon nanofibers (CNF) to improve the mechanical, electrical, and thermal properties of polymers. In recent years, carbon nanotubes have been used to improve electrical and mechanical properties of polymers (Anazawa, 2002; Choi, 2007; Du, 2004; Gao, 2007; Park, 2007; Moniruzzaman, 2006). However, by some reason of the advantage, provided by surface morphology, the literature focused on polymer composite thin films (Andrews, 2002; Bin, 2006; Blanchet, 2003; Hill, 2002; Kymakis, 2002; Shaffer, 1999; Qian, 2000) while there are no detailed studies on tri-dimensional composite gels with carbon nanotube content. Recently, carbon nanotubes and their polymer composites are used in various industrial areas such as flat panel screens, electron microscope guns, gas discharge tubes, microwave amplifiers, fuel cells, batteries, hydrogen storing media, nano probes, sensors and body-parts of aircrafts and spacecrafts (Ajayan, 2001). Some CNTs are stronger than steel and lighter than aluminum and more conductive than copper (Ajayan, 2001). Thus, studies on polymer-CNT composites have been accelerated at last decade.

Composite gels appear during a random linking process of monomers to larger and larger molecules. Even though the sol-gel transition is not a phase transition in thermodynamic sense, being a geometrical one, as a subject of critical phenomenon, it behaves like a second order phase transition and constitutes a universal class by itself (Tanaka, 1981). Experimental techniques used for monitoring sol-gel transition must be very sensitive to the structural changes, and should not disturb the system mechanically. Fluorescence technique is of particularly useful for elucidation of detailed structural aspects of the gels. This technique is based on the interpretation of the change in anisotropy, emission and/or excitation spectra, emission intensity, and viewing the lifetimes of injected aromatic molecules to monitor the change in their microenvironment (Barrow, 1962; Birks, 1970; Herculus, 1965; Galanin, 1995).

Electrical measurements are an unambiguous criterion of the existence of a percolated network in the case of conductive fillers in an isolating matrix. Dielectric measurements performed with varying frequency can lead to additional information about the percolation network as it was shown for percolation structures of carbon black in polymeric matrices. Recently, results on percolated structures of carbon nanotubes in disc sheet dry gels were presented (Pötschke, 2004). Similarly, the AC and DC conductivities of carbon nanotubespolyepoxy composites have been investigated from 20 to $110^{\circ} \mathrm{C}$ in the frequency range $10^{-2}$ 
$10^{6} \mathrm{~Hz}$ as a function of the conductive weight fraction, $p$ ranging from 0.04 to $2.5 \mathrm{wt} \%$ (Barrau, 2003). The ability of existing theories to predict electrical properties of conductive fiber composites have been examined (Weber, 1997). Complex permittivity and related AC conductivity measurements in the frequency range between $10^{-4}-10^{7} \mathrm{~Hz}$ are presented for composites of polycarbonates (PC) filled with different amounts of multiwalled carbon nanotubes (MWNTs) varying in the range between 0.5 and $5 \mathrm{wt} \%$ (Pötschke, 2003). In addition, the conductivity of dry gel composites such as polystyrene (Chang, 2006) and polypropylene (Seo, 2004) has been enhanced significantly with the addition of CNTs. On the other hand, the PAM -CNTs composite thin films was characterized by the instruments of Fourier transform infrared spectroscopy, UV absorbance spectra, fluorescence spectra and transmission electron microscope $(\mathrm{Li}, 2004)$ Dielectric spectroscopy represents a method which has been successfully applied to investigate the percolation structure of CNTs in polymer (see, e.g.(Chang, 2006; Hu, 2006; Seo, 2004)). Therefore, we expect that this method can lead to new insights in MWNTs doped systems.

This work initially aims to study the influence of MWNTs dispersion on gelation (occurring composite gels) and conductivity properties of composites. In this work, when polymer systems which are initially of isolator character doped with carbon nanotubes of nano dimensions and when amount of such addition exceeds a critical value known as percolation threshold, composite gel systems with carbon nanotube addition capable of electrically converting into conductor structure are obtained. Composite gels are analyzed using the steady state fluorescence and dielectric spectroscopy. Here the total monomer concentration is kept $2 \mathrm{M}$ and very small amount of pyranine added to the prepolymerization solution presented a spectral shift to the shorter wavelengths upon the initiation of polymerization. This spectral shift is due to the binding of pyranine to the polymer chains during the formation of PAM-MWNTs composite gels. The pyranine, thus, becomes an intrinsic fluoroprobe while it is extrinsic at the beginning of the reaction. The fluorescence intensity of the pyranine bonded to the strands of the polymers allows one to measure directly the gel fraction near the sol-gel phase transition, and thus the corresponding critical exponent, $\beta$.

Finally this work is designed to investigate the percolation structure of conductivity, and the state of dispersion of MWNTs in polyacrylamide using dielectric spectroscopy. Here critical conductivity exponent, around the conductivity percolation threshold where a material shifts to a conductor from an insulator character, is determined and found to be about 2.0 in agreement with random resistor network.

\section{Theoretical considerations}

\subsection{Conductivity percolation}

It has been established the percolation theory has been used to interpret the behavior in a mixture of conducting and non conducting components (Weber, 1997). Schematic representation of electrical percolation threshold (before and after) is shown Figure 1. Direct connection and overlapping of the CNT is not necessary i.e. nanotubes do not need to physically touch each other for conductivity. Nanotubes can just be close enough to allow for a hopping/ tunneling electron effect; these mechanisms require the CNT- CNT distance to be less than $5 \mathrm{~nm}$. As a result, a higher volume fraction of CNTs filler is needed to achieve electrical percolation threshold (Hu, 2006). Two classes of models have been developed for the description of the frequency dependence of the complex conductivity 


$$
\sigma^{*}(w)=\sigma^{\prime}(w)-i \sigma^{\prime \prime}(w)
$$

where $\sigma^{`}(w)$ real part, $\sigma^{\prime \prime}(w)$ imaginary part of the complex conductivity, $i$ imaginary unit, and $w$, angular frequency in percolating systems:

- the equivalent circuit model which treats a percolation system as a random mixture of resistors and capacitors(Bergman, 1977; Stauffer, 1994) and (ii) model based on charge carrier diffusion on percolating clusters (Sahimi, 1994; Stauffer 1994) For twocomponent systems- percolation cluster of high conductivity $\left(\sigma_{1}\right)$ embedded in a matrix of considerable lower conductivity $\left(\sigma_{2}, \sigma_{2} \ll \sigma_{1}\right)$

- macroscopic effective conductivity near the percolation threshold $p_{c}$ can be written in the scaling form (Bergman, 1977; Stauffer, 1994; Straley, 1977).

$$
\sigma(p, w) \alpha \sigma_{1}\left|p^{\prime}-p_{c}^{\prime}\right|^{r} \Phi_{ \pm}\left(\frac{\sigma_{2}}{\sigma_{1}}\left|p^{\prime}-p_{c}^{\prime}\right|^{-(r+s)}\right)
$$

where $p^{\prime}$ is the concentration of conducting filler, $\Phi_{+}$and $\Phi_{-}$denote scaling functions for $p^{\prime}>p_{c}^{\prime}$ and $p^{\prime}<p_{c}^{\prime}$ respectively, $s$ and $r$ are the critical exponents.
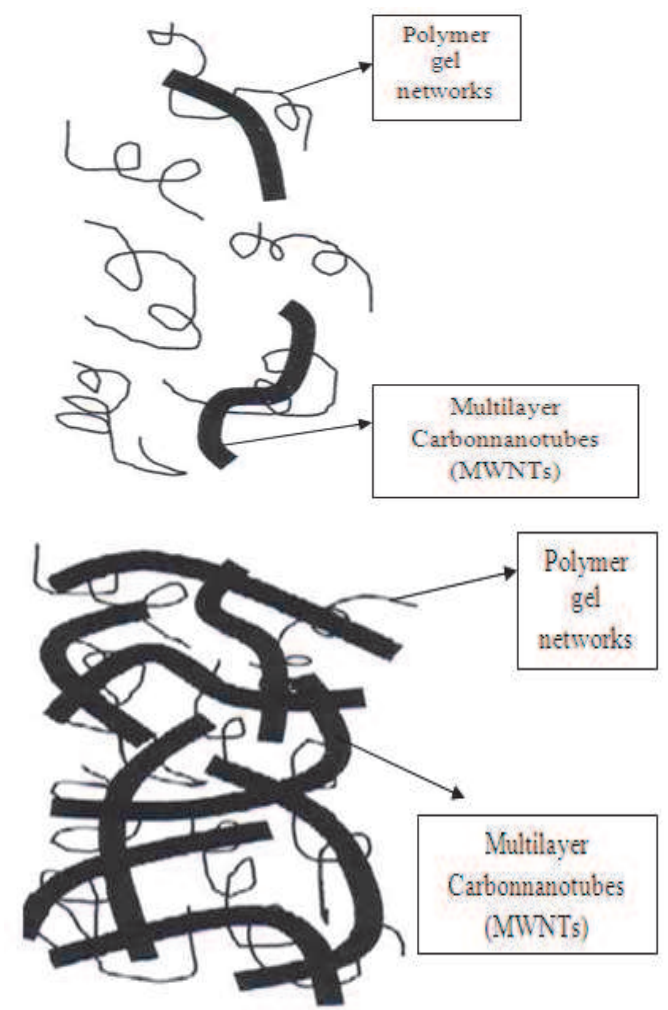

Fig. 1. Schematic of MWNTs/gel composite, before and after the gel formation with isotropic orientation of nanotubes. 
These models treat a percolation system as a random mixture of resistors, $R$ and capacitors $C$ having a characteristic relaxation time $\tau=\frac{1}{w_{0}}=R C$, scaling law Eq. 2 for the complex conductivity near the percolation threshold takes the form (Bergman, 1977; Pearson, 1978; Seo, 2004)

$$
\sigma^{*}(p, w) \alpha \frac{1}{R}\left|p^{\prime}-p_{c}^{\prime}\right|^{r} \Phi_{ \pm}\left(\frac{i w}{w_{0}}\left|p^{\prime}-p_{c}^{\prime}\right|^{-(r+s)}\right)
$$

One of the main physical content of this key scaling law is the existence of a time scale

$$
\tau_{c}\left(p^{\prime}\right) \alpha \frac{1}{\omega_{p}\left(p^{\prime}\right)} \alpha \frac{1}{\omega_{0}}\left|p^{\prime}-p_{c}^{\prime}\right|^{-(r+s)}
$$

that diverges as the percolation threshold is approached from both sides.

For the frequency dependence of AC conductivity at the percolation threshold Bergman and Imry (Bergman, 1977) derived the following law:

$$
\sigma^{\prime}(w) \alpha w^{\frac{r}{r+s}}
$$

where $\sigma^{\prime}$, the real part of Eq.1; $w$, angular frequency; $\mathrm{r}$ and $\mathrm{s}$ critical exponents for conductivity. A wide variety of approaches have been applied to the determination of these exponents as given in Table 1 for 3D with references (Adler, 1990; Bergman, 1977; Derrida, 1983; Fisch, 1978; Gingold, 1990; Herrman, 1984; Sahimi, 1994; Straley, 1977).

\begin{tabular}{cccc}
\hline System & $r$ & $s$ & References \\
\hline Resistor lattice & $1.70 \pm 0.05$ & $0.70 \pm 0.05$ & Straley, 1977 \\
Random resistor network & $1.95 \pm 0.03$ & - & Fish and Harris, 1978 \\
Random resistor network & $2.02 \pm 0.05$ & - & Adler, 1990 \\
Random resistor lattices & $2.003 \pm 0.047$ & - & Gingold and Lobb, 1990 \\
& & & Herrman, 1984 \\
Random resistor network & $1.9 \pm 0.1$ & $0.75 \pm 0.04$ & Derrida, 1983 \\
\hline
\end{tabular}

Table 1. Critical exponents for electrical percolation threshold in three dimension (3D).

\subsection{Sol-gel phase transition}

In the past half century various models have been developed to describe gel formation, among which Flory-Stockmayer theory and percolation theory provide bases for modeling the sol-gel phase transition (Flory, 1941; de Gennes, 1988; Hermann, 1986; Stauffer, 1982, 1985; Stockmayer, 1943). Statistical theories based on tree-like structure (Bethe lattice) uses mean field approximation, originate from Flory (Flory, 1941) and Stockmayer (Stockmayer, 1943), and assume equal reactivities of functional groups and the absence of cyclization reactions. Most statistical theories derived in the following decades are fully equivalent, differing only in mathematical language (Durand, 1979; Gordon, 1962; Macosko, 1976; Pearson, 1978). 
Percolation offers a particularly simple and yet detailed picture in terms of which one may seek to understand gelation (de Gennes, 1988; Stauffer, 1985, 1994). In the language of percolation, one may think of monomers as occupying the vertices of a periodic lattice, and the chemical bonds as corresponding to the edges joining these vertices at any given moment, with some probability, $p$. Then, the gel point can be identified with the percolation threshold $p_{c}$, where, in the thermodynamic limit, the incipient infinite cluster starts to form. Identifying the weight average degree of polymerization $D P_{w}$ with the average cluster size $S_{a v}$ and the gel fraction $G$ with the probability $P_{\infty}$ of an occupied site to belong to the incipient infinite cluster, one can predict the scaling behavior of these and related quantities near the gel point, as a function of $\left|p-p_{c}\right|$,

$$
\begin{array}{ll}
D P_{w} \propto\left(p_{c}-p\right)^{-\gamma} & p \rightarrow p_{c^{-}} \\
G \propto\left(p-p_{c}\right)^{\beta} & p \rightarrow p_{c^{+}}
\end{array}
$$

If $\mathrm{p}$ approaches $p_{c}$ from below it is denoted as $p \rightarrow p_{c}{ }^{-}$, in contrast, $p \rightarrow p_{c}{ }^{+}$denotes when $p$ approaches $p_{c}$ from above. Here, $\beta$ and $\gamma$ are the critical exponents, respectively. The critical exponents in percolation theory, $\beta=0.41$ and $\gamma=1.70$, differ from those found in Flory-Stockmayer, $\beta=1$ and $\gamma=1$.

\section{Experimental}

PAM- MWNT composites were prepared from various amounts of AAm and MWNTs. The solution is composed of MWNTs (Cheap Tubes Inc.), Polyvinyl pyrolidone (PVP), and water. The AAm and MWNTs concentrations are given in (Table 2). BIS (Merck) dissolving in $40 \times 10^{-6} \mathrm{~m}^{3}$ of water in which $10^{-4} 1 \mathrm{t}$ of TEMED (tetramethylethylenediamine) was added as an accelerator. The initiator, Ammonium persulfate (APS, Merck) was used and the initiator and pyranine concentrations were kept constant at $7 \times 10^{-3} \mathrm{M}$ and $4 \times 10^{-4} \mathrm{M}$, respectively, for all samples. All samples were deoxygenated by bubbling nitrogen for 10 minutes, just before gelation process has started.

The Model LS-50 spectrometer of Perkin-Elmer was used for the fluorescence intensity measurements. All measurements were made at $90^{\circ}$ position and slit widths were kept at 5 $\mathrm{nm}$. Pyranine was excited at $340 \mathrm{~nm}$ during in situ gelation experiments and variation in the fluorescence spectra and emission intensity of the pyranine were monitored as a function of gelation time.

The AC conductivity measurements were carried out by means of an HP- 4192 A impedance analyzer controlling a computer at room temperature. The dielectric cell was a parallel capacitor with sample thickness of $2 \mathrm{~mm}$.

\section{Results and discussion}

\subsection{Conductivity exponents}

The conductivity as a function of alternative current frequency is shown in Figure 2 for the gels with different content of PAM and/or MWNTs. It is observed that conductivity 
behavior of the composite gels increased exponentially by increasing AC frequency. The results show that the critical conductivity behavior of the composite system occurs at $4 \mathrm{kHz}$ for lower MWNTs content while this behavior occurs nearly $0 \mathrm{kHz}$ for higher MWNTs contents. PAM doped by lower MWNTs composite system converts into conductive character at higher frequency than the frequency value needed for PAM doped by higher MWNTs composite system. From figure 2, one can seen that the conductivity, $(\sigma)$ of $0.3 \%$ MWNT gel was stable up to $4 \mathrm{kHz}$ then suddenly increased to some point, and become stable. On the other hand, the conductivity, $(\sigma)$ for 1.0 and $3.0 \%$ MWNT gels increased suddenly at very low frequency, then become stable after $4 \mathrm{kHz}$.

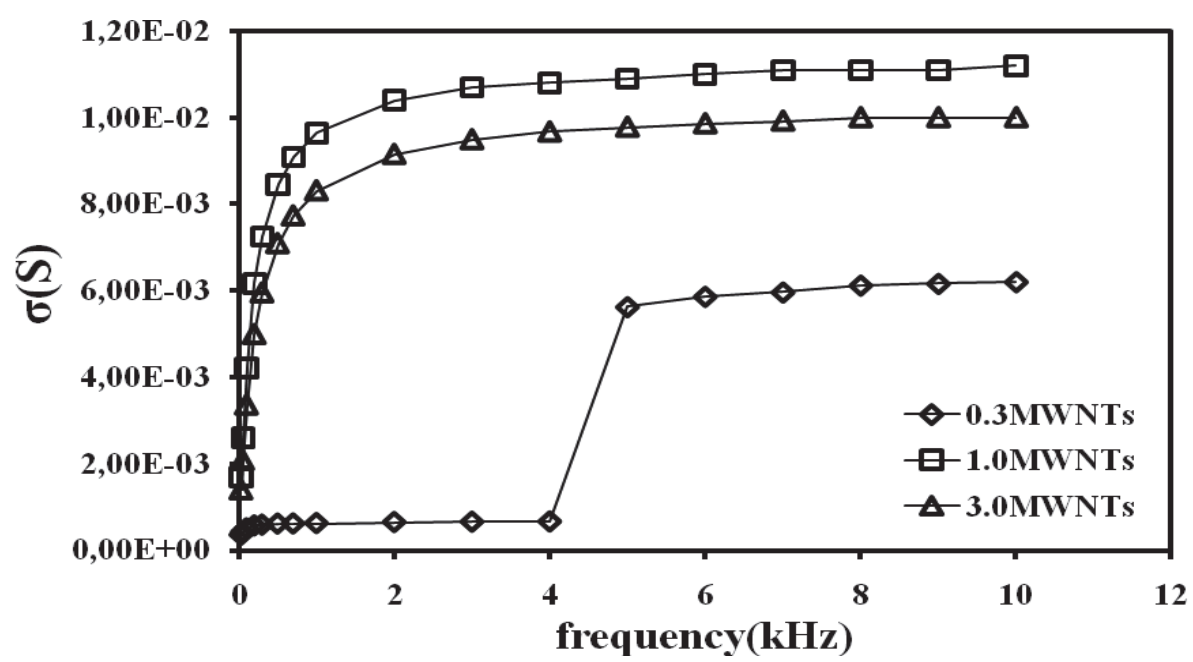

Fig. 2. Conductivity of PAM- MWNTs composites versus alternatively current (AC) frequency at room temperature for $0.3 \%$ MWNTs, $1.0 \%$ MWNTs and $3.0 \%$ MWNTs contents, respectively.

The effect of MWNTs content on conductivity of PAM- MWNTs composite gels is presented in Figure 3 for $5 \mathrm{kHz}$. At very low content of MWNTs (0.3\%), the conductivity is quite low, however, after $0.3 \%$, a definite increase in conductivity is observed. This stepwise change in conductivity is a result of the formation of an interconnected structure of MWNTs and can be regarded as an electrical percolation threshold which simply means that at contents between 0.3 and $1.0 \%$ MWNTs, some percentage of electrons are permitted to flow through the specimen due to the creation of an interconnecting conductive pathway. At contents above $1.0 \%$ MWNTs, the conductivities are stable by increasing MWNTs content (Seo, 2004).

The value of the fitting exponent $r$ in Eq. 7 was estimated from the slope of the linear relation between $\log \sigma$ and $\log w$ as shown in Figure 4. $s$ is the critical exponent which taken from literature by $s \approx 0.7 \pm 0.05$ (Straley, 1977). The produce $\mathrm{r}$ values are listed in Table 2 where it is seen that the electrical percolation occurs above $0.3 \%$ MWNTs with a critical exponent around $r \approx 2$ which is close to the theoretical prediction of this value in 3D percolated system as known random resistor network (Derrida, 1983; Straley, 1977). 


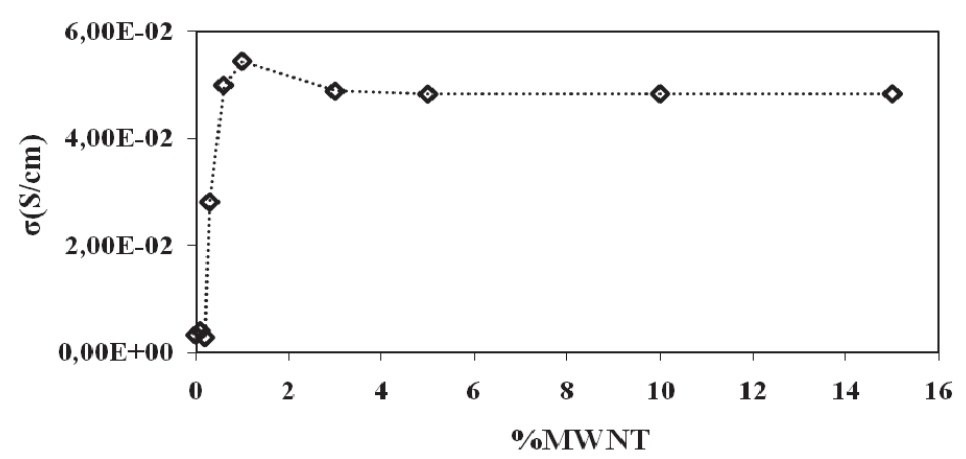

Fig. 3. Conductivity as a function MWNTs content at $5 \mathrm{kHz}$.
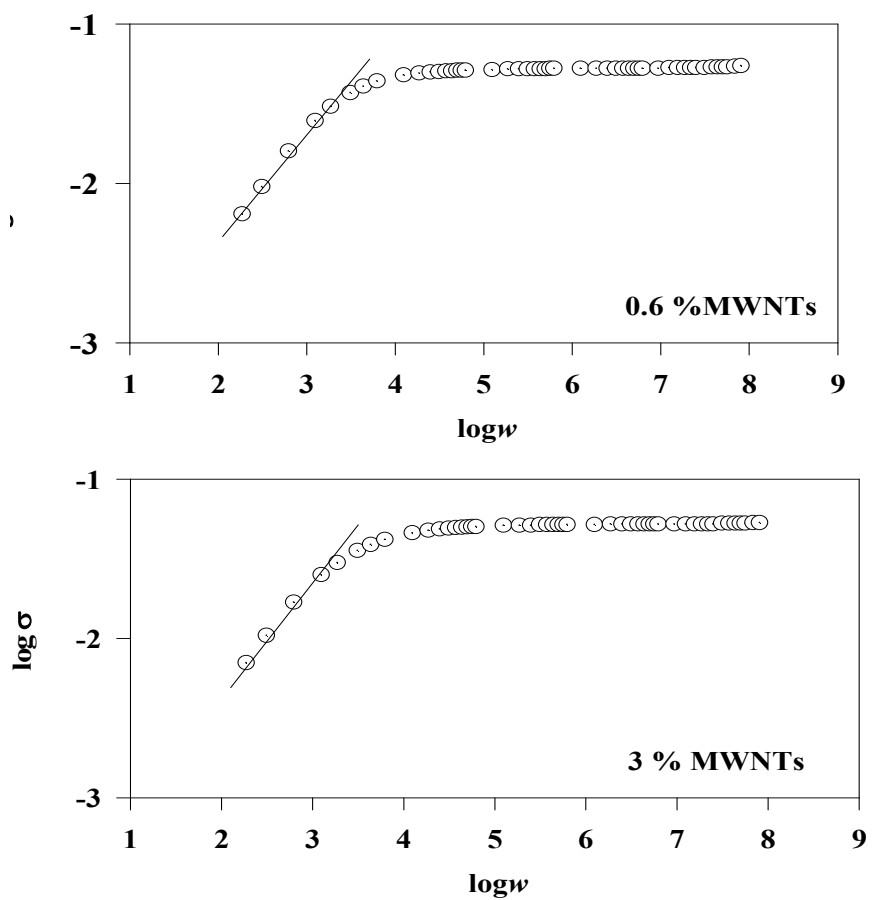

Fig. 4. Logarithmic plot of the conductivity versus frequency curves of $0.6 \%$ MWNTs and 3 $\%$ MWNTs, respectively.

\subsection{Sol-gel exponents}

Figure 5 shows the typical fluorescence spectra of pyranine at two different stages of gelation for PAM-MWNTs composite. Beginning of the reaction only the 512nm peak exists, and then the intensity of the new peak around $380 \mathrm{~nm}$ started to increase as the intensity of the 512nm peak decreased during the course of gelation of PAM-MWNTs composite gels. 

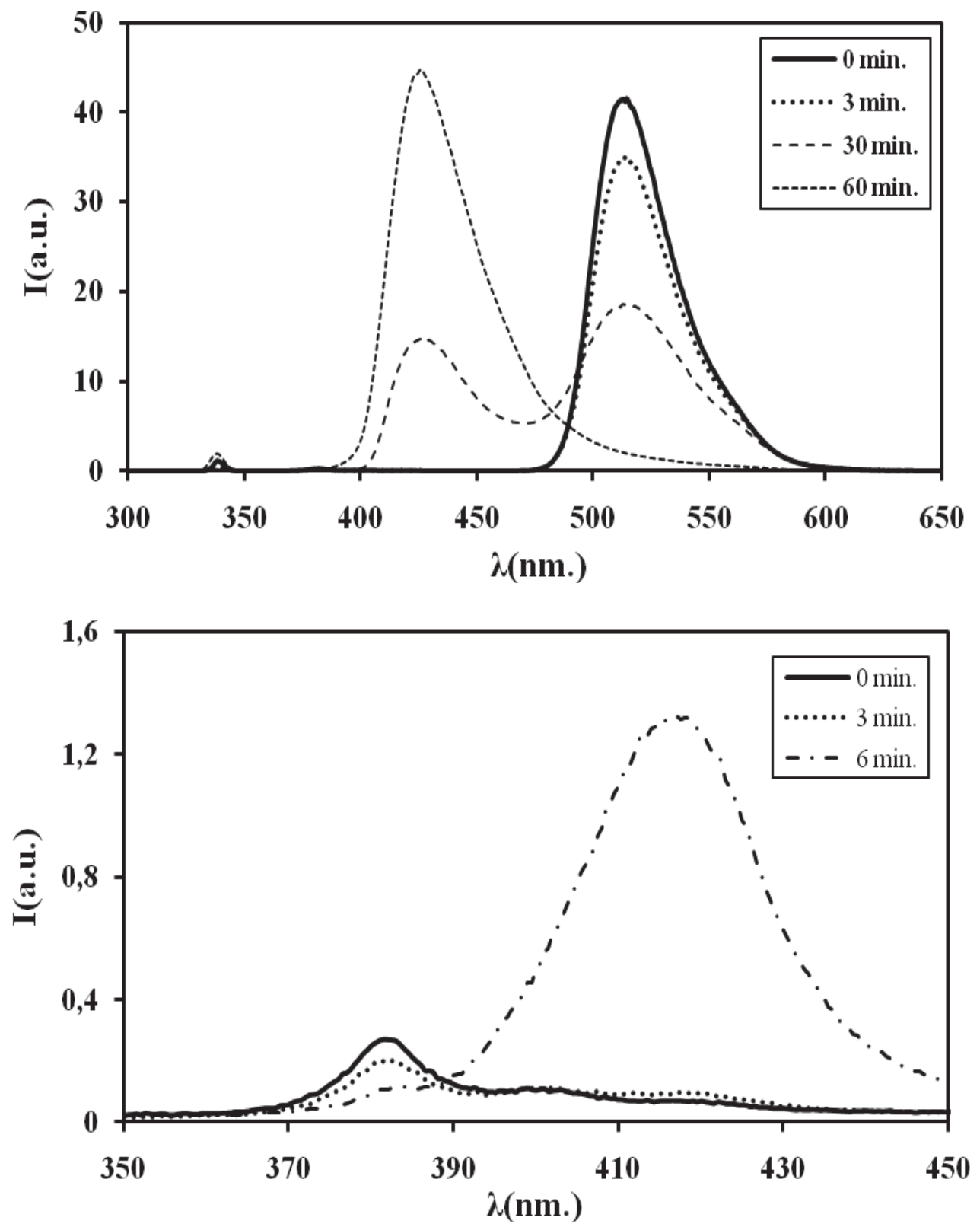

Fig. 5. Typical fluorescence spectra of pyranine at different stages of gelation for PAMMWNTs composite during the whole course of gelation presenting the binding of pyranines and at the early stage of gelation, presenting the spectral shift from $380 \mathrm{~nm}$ toward $427 \mathrm{~nm}$, respectively. 


\begin{tabular}{|c|c|c|c|c|c|}
\hline AAm (\%) & MWNTs(\%) & $t_{\text {gel }}(\mathrm{s})$ & $\mathrm{C}^{-} / \mathrm{C}^{+}$ & $\beta$ & $r$ \\
\hline & & & 1.0 & 0.92 & \\
\hline & & & 0.37 & 0.50 & \\
\hline \multirow[t]{5}{*}{100} & 0 & $300 \pm 5$ & 0.28 & 0.55 & - \\
\hline & & & 0.23 & 0.52 & \\
\hline & & & 0.1 & 0.52 & \\
\hline & & & 1.0 & 0.75 & \\
\hline & & & 0.37 & 0.50 & \\
\hline \multirow[t]{5}{*}{99.9} & 0.1 & $300 \pm 5$ & 0.28 & 0.56 & $0.10 \pm 0.02$ \\
\hline & & & 0.23 & 0.48 & \\
\hline & & & 0.1 & 0.50 & \\
\hline & & & 1.0 & 1.05 & \\
\hline & & & 0.37 & 0.50 & \\
\hline \multirow[t]{5}{*}{99.8} & 0.2 & $300 \pm 5$ & 0.28 & 0.65 & $0.57 \pm 0.02$ \\
\hline & & & 0.23 & 0.60 & \\
\hline & & & 0.1 & 0.50 & \\
\hline & & & 1.0 & 0.75 & \\
\hline & & & 0.37 & 0.66 & \\
\hline \multirow[t]{5}{*}{99.7} & 0.3 & $300 \pm 5$ & 0.28 & 0.58 & $0.34 \pm 0.02$ \\
\hline & & & 0.23 & 0.55 & \\
\hline & & & 0.1 & 0.50 & \\
\hline & & & 1.0 & 0.96 & \\
\hline & & & 0.37 & 0.67 & \\
\hline \multirow[t]{5}{*}{99.4} & 0.6 & $360 \pm 5$ & 0.28 & 0.60 & $1.80 \pm 0.02$ \\
\hline & & & 0.23 & 0.55 & \\
\hline & & & 0.1 & 0.55 & \\
\hline & & & 1.0 & 0.90 & \\
\hline & & & 0.37 & 0.65 & \\
\hline \multirow[t]{5}{*}{99} & 1 & $420 \pm 5$ & 0.28 & 0.57 & $1.90 \pm 0.02$ \\
\hline & & & 0.23 & 0.65 & \\
\hline & & & 0.1 & 0.60 & \\
\hline & & & 1.0 & 0.83 & \\
\hline & & & 0.37 & 0.51 & \\
\hline \multirow[t]{5}{*}{97} & 3 & $420 \pm 5$ & 0.28 & 0.48 & $1.90 \pm 0.02$ \\
\hline & & & 0.23 & 0.50 & \\
\hline & & & 0.1 & 0.50 & \\
\hline & & & 1.0 & 1.10 & \\
\hline & & & 0.37 & 0.70 & \\
\hline \multirow[t]{3}{*}{95} & 5 & $480 \pm 5$ & 0.28 & 0.63 & $2.30 \pm 0.08$ \\
\hline & & & 0.23 & 0.68 & \\
\hline & & & 0.1 & 0.65 & \\
\hline
\end{tabular}




\begin{tabular}{llllll}
\hline AAm $(\%)$ & MWNTs $(\%)$ & $\mathrm{t}_{\text {gel }}(\mathrm{s})$ & $\mathrm{C}^{-} / \mathrm{C}^{+}$ & $\beta$ & $r$ \\
\hline \multirow{3}{*}{10} & & 1.0 & 0.88 & \\
90 & \multirow{3}{*}{$540 \pm 5$} & 0.37 & 0.72 & \\
& & 0.28 & 0.57 & $1.80 \pm 0.05$ \\
& & 0.23 & 0.60 & \\
& & 0.1 & 0.56 & \\
& & 1.0 & 1.10 & \\
& & & 0.37 & 0.45 & \\
& \multirow{3}{*}{15} & \multirow{2}{*}{$660 \pm 5$} & 0.28 & 0.40 & $1.90 \pm 0.02$ \\
& & 0.23 & 0.41 & \\
& & 0.1 & 0.40 & \\
\hline
\end{tabular}

Table 2. Experimentally measured parameters for PAM-MWNTs composite gels.

As one can see from Figure 6 the intensity of the short-wavelength peak (380 nm-peak) increases during the course of polymerization, meanwhile as the polymerization progresses the maxima of the short wavelength-peak shifts to some higher wavelengths from $380 \mathrm{~nm}$ to 427nm (Kaya et al., 2004). Here at first the shift from $512 \mathrm{~nm}$ to $380 \mathrm{~nm}$ in the emission spectra due to a $\mathrm{C}$ - $\mathrm{O}$ ether bond formation between the hydroxyl oxygen of $3 \mathrm{sPyOH}$ and a terminal $\mathrm{C}$-atom of the growing AAm chain.

Then the shift in the short-wavelength peak between 380 and $427 \mathrm{~nm}$ is probably due to the complexation of $\mathrm{SO}_{3}^{-}$groups with protonated amide groups whether on the same polymer molecule or on the other polymer strands (Y1lmaz et al., 2009). The reason for the shift in the isoemissive (isostilbic) point is the change in the internal morphology of the system: at the beginning of the polymerization the system is in the "sol" state (all pyranine are free) and above a certain time it turns into the "gel" state (most of pyranine are bonded).

Figure 6 presents the fluorescence intensity, $\mathrm{I}_{512}$ of the free pyranine $(512 \mathrm{~nm}$.) from the reaction mixture as a function of the gelation time for 1 and $5 \%$ MWNTs concentrations. As seen in Figure 6 that the fluorescence intensity increased up to some point (gelation stage), and then decreased to zero at the end of the reaction (final stage) for different MWNTs concentration.

Figure 7 presents the fluorescence intensities, $I_{e m}$ from the bonded pyranine (427nm) against the gelation time for 1 and $5 \%$ MWNTs concentrations. The maxima of the spectra shift from $380 \mathrm{~nm}$ to $427 \mathrm{~nm}$ as the gelation progresses. Therefore, we monitored the fluorescence spectra in relatively large periods of time and plotted the intensity $I_{e m}$ corresponding to the maxima of the spectra as a function of time. These data were used to evaluate the critical behavior of the sol- gel phase transition (Kaya et al., 2004).

For the determination of the gel points, $t_{\text {gel }}$, each experiment was repeated at the same experimental conditions, and the gel points were produced by dilatometric technique (Okay et al., 1999). A steel sphere of $4.8 \mathrm{~mm}$ diameter was moved in the sample up and down slowly by means of a piece of magnet applied from the outer face of the sample cell. The time at which the motion of the sphere is stopped was evaluated the onset of the gel point, $t_{\text {gel }}$. The $t_{g e l}$ values are summarized in Table 2 together with the other parameters.

Now, it can be argued that the total fluorescence intensity from the bonded pyranines monitors the weight average degree of polymerization and the growing gel fraction for below and above the gel point, respectively. This proportionality can easily be proven by 
using a Stauffer type argument under the assumption that the monomers occupy the sites of an imaginary periodic lattice (Stauffer et al., 1982; 1985; 1994).

Gelation theory often makes the assumption that the conversion factor, $p$, alone determines the behavior of the gelation process, though $p$ may depend on temperature, concentration of monomers, and time (Stauffer et al. 1982; 1994). If the temperature and concentration are kept fixed, then $p$ will be directly proportional to the reaction time, $t$. This proportionality is not linear over the whole range of reaction time, but it can be assumed that in the critical region, i.e. around the critical point $\left|p-p_{c}\right|$ is linearly proportional to the $\left|t-t_{\text {gel }}\right|$ (Yilmaz et al., 1998, 2002). Therefore, below the gel point, i.e., for $t<t_{\text {gel }}$ the maximum fluorescence intensity, $I$, measures the weight average degree of polymers (or average cluster size). Above $t_{g e l}$, if the intensity from finite clusters distributed through the infinite network $I_{c t}$ is subtracted from the maximum fluorescence intensity, then, the corrected intensity $I-I_{c t}$ measures solely the gel fraction $G$, the fraction of the monomers that belong to the macroscopic network. In summary, we have the following relations, (Kaya et al., 2004)

$$
\begin{array}{ll}
I^{\prime} \propto D P_{w}=C^{+}\left(t_{g e l}-t\right)^{-\gamma} & t \rightarrow t_{g e l}^{-} \\
I_{c t} \propto D P_{w}=C^{-}\left(t_{g e l}-t\right)^{-\gamma^{\prime}} & t \rightarrow t_{g e l}{ }^{+} \\
I^{\prime}-I_{c t} \propto G=B\left(t-t_{g e l}\right)^{\beta} & t \rightarrow t_{g e l^{+}}
\end{array}
$$

where $C^{+}, C^{-}$and $B$ are the critical amplitude, respectively.

It has been well established that the average cluster size of the finite clusters (distributed through the infinite network) above the gel point decreases with the same, but negative slope of the increasing cluster size before the gel point. This means that the exponents $\gamma$ and $\gamma^{\prime}$, defined for the cluster sizes below and above the gel point, have the same values (de Gennes, 1988; Hermann, 1986; Stauffer et al., 1982, 1985, 1994). But, the critical amplitudes for the average cluster size defined below $\left(C^{+}\right.$in Eq. 8a) and above $\left(C^{-}\right.$in Eq. 8 b) the gel point are different, and there exist a universal value for the ratio $C^{+} / C^{-}$. This ratio is different for mean-field versus percolation as discussed by Aharony (Aharony, 1980) and Stauffer (Stauffer, 1982). The estimated values for $C^{+} / C^{-}$(Stauffer, 1982, Aharony, 1980) is given in Table 3. In order to determine the intensity $I_{c t}$ in Eqs.(8b) and (9), we first choose the parts of the intensity-time curves up to the gel points, then the mirror symmetry $I_{m s}$ of this parts according to the axis perpendicular to time axis at the gel point were multiplied by the ratio $C^{-} / C^{+}$, so that $I_{c t}=\frac{C^{-}}{C^{+}} I_{m s}$. Thus, $I_{c t}$ measures solely the intensity from the cluster above the gel point, and $I^{\prime}-I_{c t}$ measures the intensity from the gel fraction. This process is clarified explicitly in Figure 8.

Using the Eq. (9), and the values for $t_{g e l}$ summarized in Table 2 we calculated $\beta$ exponents as a function of AAm and/or MWNTs contents. Figure 9 represents the log-log plots of the typical intensity- time data above the gel point, for $0.6 \%$ MWNTs and $1 \%$ MWNTs concentration, respectively, where the slope produced $\beta$ values which are listed in Table 2 for various PAM-MWNTs. 

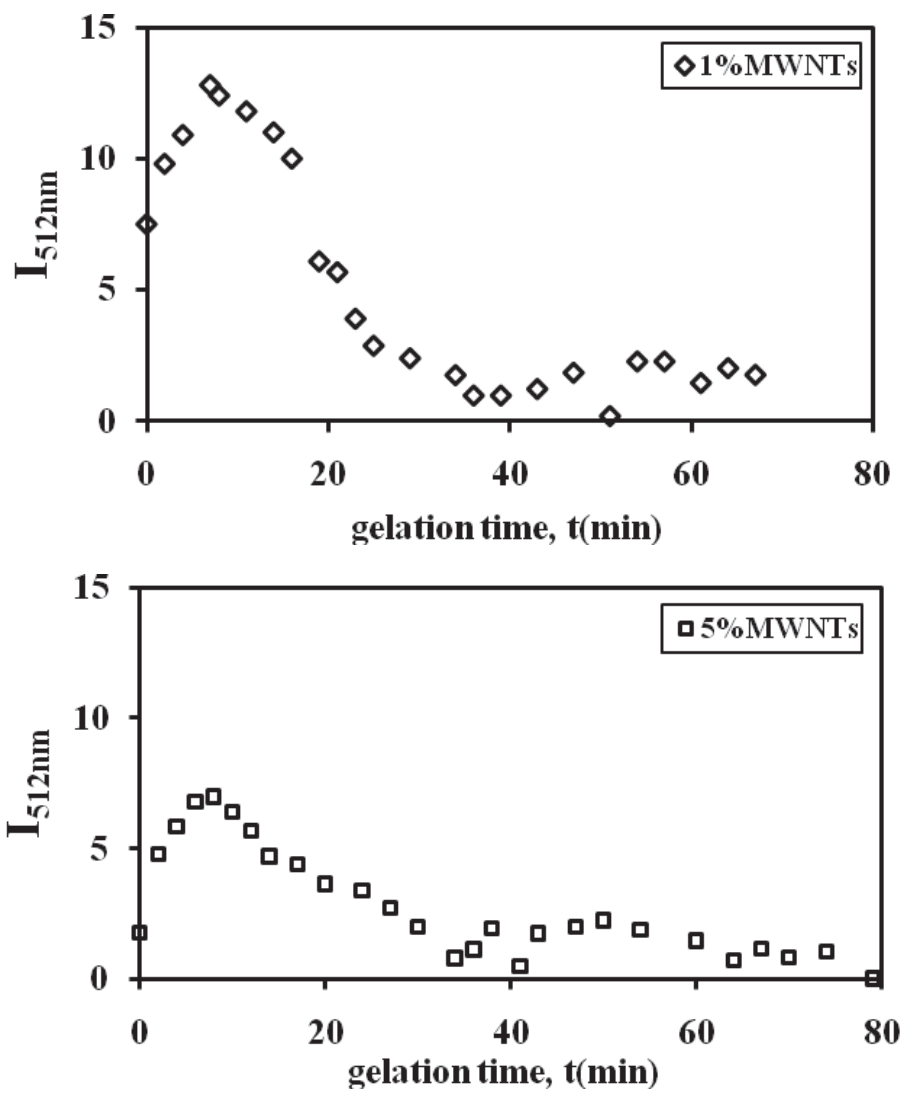

Fig. 6. Fluorescence intensity of the free pyranine at $512 \mathrm{~nm}, \mathrm{I}_{512 \mathrm{~nm}}$, versus reaction time for 1 $\%$ MWNTs and $5 \%$ MWNTs contents.

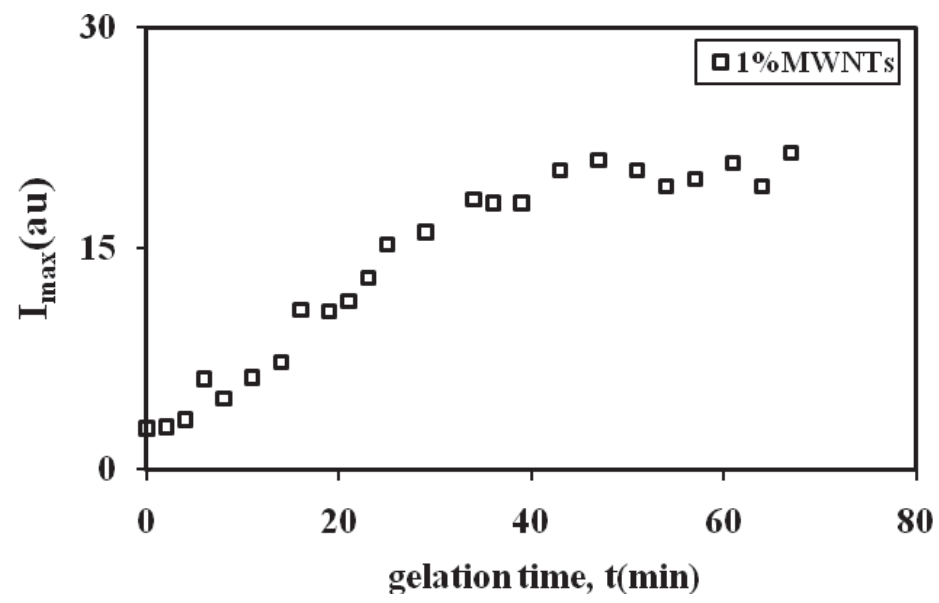




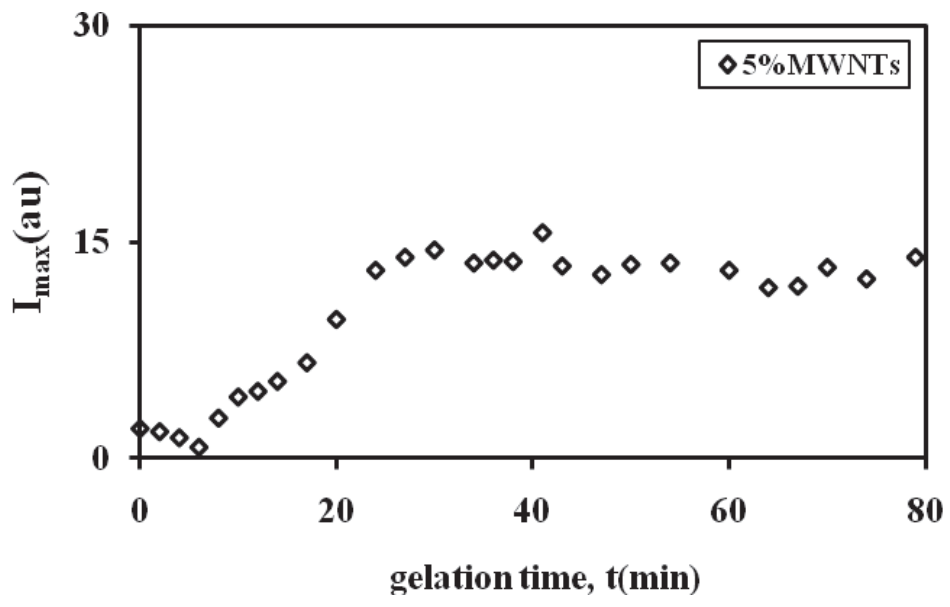

Fig. 7. Fluorescence intensity $427 \mathrm{~nm}$. variation of the pyranine, bonded to the PAM for different MWNTs concentrations, versus reaction time for $1 \% \mathrm{MWNTs}$ and $5 \% \mathrm{MWNTs}$ contents, respectively.

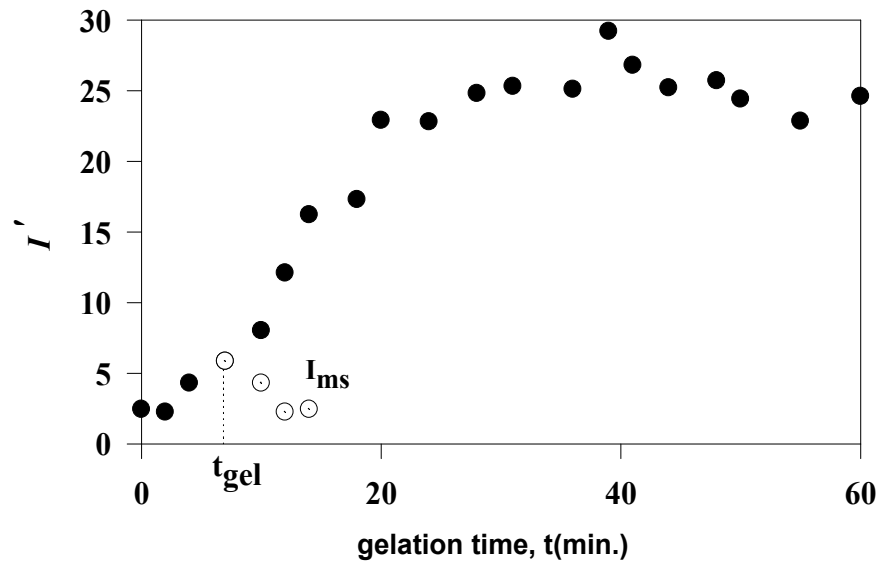

Fig. 8. Intensity- time curve during occurring of PAM- $0.6 \%$ MWNTs composite gels. The curve depicted by dots represents the mirror symmetry $I_{m s}$ of the intensity according to the axis perpendicular to time axis at $t=t_{\text {gel. }}$. The intensity from the clusters above the gel point is calculated as $I_{c t}=\frac{C^{-}}{C^{+}} I_{m s}$. Thus, $I-I_{c t}$ monitors the growing gel fraction for $t>t_{g e l}$. The intensity from the below part of the symmetry axis monitor the average cluster size for $t<t_{g e l}$. 


\begin{tabular}{cccccc}
\hline & Classical & \multicolumn{4}{c}{ Percolation } \\
\cline { 2 - 6 } & $\begin{array}{c}\text { Direct } \\
\varepsilon \text { expansion }\end{array}$ & $\begin{array}{c}\gamma_{\text {gexp }}=1.840 \text { and } \\
\beta_{\varepsilon \exp }=0.52\end{array}$ & $\begin{array}{c}\gamma=1.7 \text { and } \\
\beta=0.4\end{array}$ & $\begin{array}{c}\text { Series and } \\
\text { Montecarlo }\end{array}$ \\
\hline$C^{-} / C^{+}$ & 1 & $1 / 2.7$ & $1 / 3.5$ & $1 / 4.3$ & $1 / 10$ \\
\hline
\end{tabular}

Table 3. The estimated values for the ratio $C^{-} / C^{+}$(Aharony, 1980)
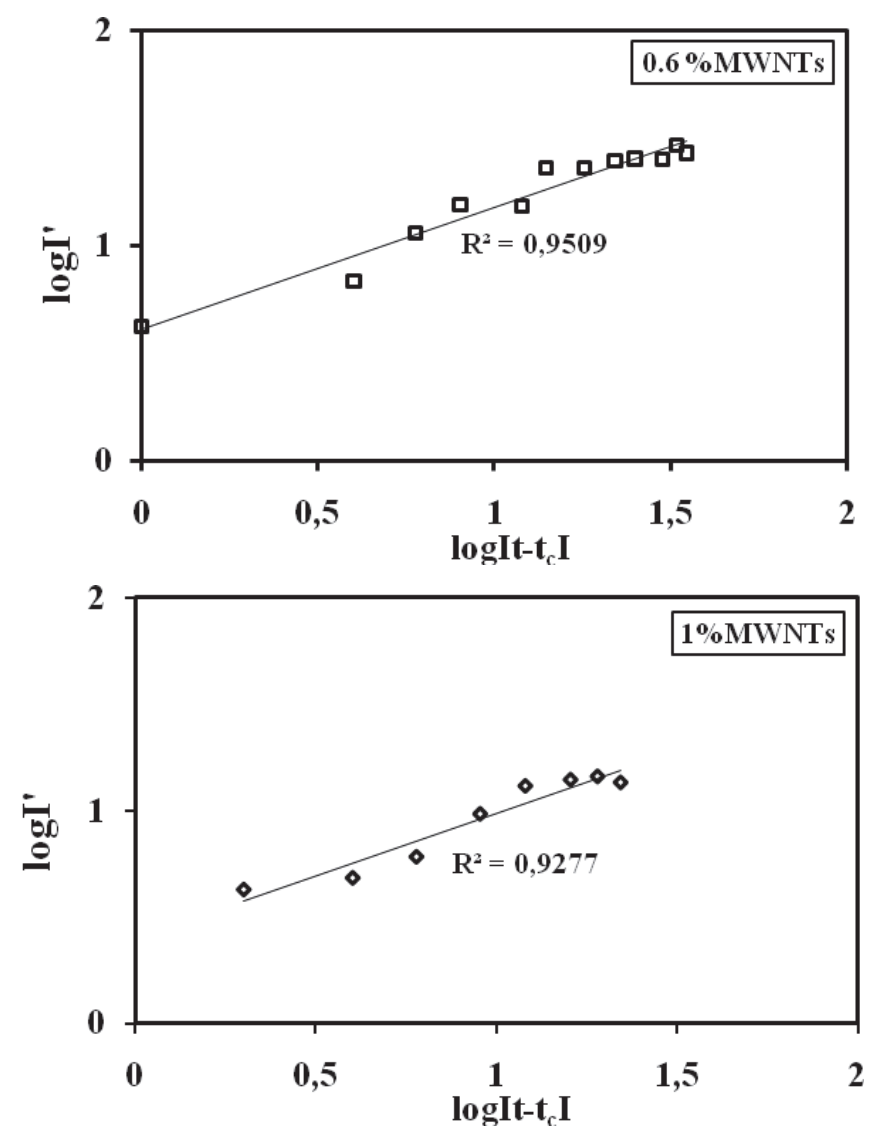

Fig. 9. Double logarithmic plot of the intensity $I$ ` versus time curves above $t_{\text {gel }}$ for 0.6 $\% \mathrm{MWNTs}$ and $1 \%$ MWNTs concentrations, respectively. The $\beta$ exponents were determined from the slope of the straight lines.

\section{Conclusion}

In conclusion, PAM- MWNTs composites were prepared by the free-radical crosslinking copolymerization. The gelation and conductivity measurement of PAM- MWNTs composites were characterized by steady state fluorescence spectroscopy and dielectric spectroscopy, respectively. Here the critical phenomena of the gelation and conductivity were tested as a function of MWNTs concentration. 
It is found that the electrical percolation threshold occurs above $0.3 \%$ MWNTs with critical exponent of $r=1.8-2.3$ which is quite close to the theoretical prediction of this value in $3 \mathrm{D}$ percolated system $(r=2)$. The percolation critical exponent, $r$ obtained from fitting the composition dependence of the AC conductivity above the percolation threshold is consistent with the suggestions of percolation for a random resistor network. It is observed that if polymer systems which are initially of isolator character doped with carbon nanotubes of nano dimensions and when amount of such addition exceeds a critical value known as percolation threshold, then composite gel systems with carbon nanotube addition became capable of electrically converting into conductor structure are obtained.

It is understood that the gel fraction exponent $\beta$ obeyed the percolation theory for PAMMWNTs composite gels. We were, thus, able to measure the copolymerization kinetics obeying the percolation picture without disturbing the system mechanically. In the mean time the universality of the sol-gel transition was tested as a function of parameters like MWNTs concentration ratios.

\section{References}

Adler, J.; Meir, Y.; Aharony, A.; Harris, A. B. \& Klein, L. (1990). Low concentration series in general dimension. Journal of Statistical Physics, Vol. 58, pp. 511- 538.

Aharony, A. (1980). Universal critical amplitude ratios for percolation, Physical Review B. Vol. 22, pp. 400-414.

Ajayan, P. M. (1994). Aligned Carbon Nanotube Arrays Formed by Cutting a Polymer Resin-Nanotube Composite, Science, Vol. 265, pp. 1212- 1214.

Ajayan, P. M. \& Zhou, O. Z. (2001). Applications of carbon nanotubes. Carbon Nanotubes (Topics in Applied Physics), ed Dresselhaus, M.S.; Avouris, Ph. Vol. 80, pp. 391425, Berlin: Springer Verlag.

Anazawa, K.; Shimotani, K.; Manabe, C.; Watanabe, H. \& Shimizu, M. (2002). High- purity carbon nanotubes synthesis method by an arc discharhing in magnetic field. Applied Physics Letters, Vol. 81, pp. 739-741.

Andrews, R.; Jacques, D.; Qian, D. \& Rantell, T. (2002) Multiwall Carbon Nanotubes: Synthesis and Application, Accounts of Chemical Research, Vol.35, pp.1008-1017.

Barrau, S.; Demont, P.; Peigney, A.; Laurent, C. \& Lacabanne, C. (2003). DC and AC Conductivity of Carbon Nanotubes- polyepoxy Composites. Macromolecules, Vol. 36, pp. 5187- 5194.

Barrow, G. M. (1962). Introduction to Molecular Spectroscopy, McGraw-Hill, New York.

Bauhofer, W. \& Kovacs, J. Z. (2009). Composite Science and Technology, Vol. 69, pp. 1486- 1498.

Bergman, D. J. \& Imry, Y. (1977).Critical Behavior of the Complex Dielectric Constant near the percolation threshold of a heterogeneous material. Physical Review Letters, Vol. 39(19), pp.1222- 1225.

Bin, Y.; Mine, M.; Koganemaru; A.; Jiang, X.; Matsuo, M. (2006).Morphology and mechanical and electrical properties of oriented PVA- VGCF and PVA- MWNT composites, Polymer, Vol.47, pp.1308-1317.

Birks, J. B. (1970). Photophysics of Aromatic Molecules, Wiley Interscience, London.

Blanchet, G. B.; Fincher, C. R. \& Gao, F. (2003). Polyaniline nanotube composites: A highresolution printable conductor, Applied Physics Letters, Vol. 82(8), pp.1290-1292.

Chang, T.-E.; Kisliuk, A.; Rhodes, S. M.; Britain, W. J.; Sokolov, A. P. (2006).Conductivity and mechanical properties of well- dispersed single wall carbon nanotube/polystyrene composite. Polymer, Vol. 47, pp. 7740- 7746. 
Choi, C. S.; Park, B.J. \& Choi, H.J. (2007). Electrical and rheological characteristics of poly (vinyl acetate)/multi-walled carbon nanotube nanocomposites, Diamond and Related Materials Vol. 16, pp. 1170- 1173.

de Gennes, P. G. (1988). Scaling Concepts in Polymer Physics, Cornell University Press, Ithaca.

Derrida, B.; Stauffer, D.; Herrman, H. J. \& Vannimenus J. (1983).Transfer matrix calculation of conductivity in three- dimensional random resistor networks at percolation threshold. Le Journal de Physics Letters, Vol. 44, pp. L701-L706.

Du, F.; Scogna, R.C.; Zhou, W.; Brand, S.; Fischer, J. E. \& Winey, K. I. (2004). Nanotube networks in polymer nanocomposites: rheology and electrical conductivity, Macromolecules, Vol. 37, pp. 9048-9055.

Durand, D. \& Bruneau, C. -M. (1979). Reactivity and Gelation. I. Intrinsic Reactivity. Journal of Polymer Science: Polymer Physics Education, Vol. 17, pp. 273- 294.

Fisch, R. \& Harris, A. B. (1978). Critical behavior of random resistor networks near the percolation threshold. Physical Review B, Vol. 18, pp. 416- 420.

Flory, P. J. (1941). Molecular Size Distribution in Three Dimensional Polymers. I. Gelation. Journal of American Chemical Society, Vol. 63, pp. 3083-3090.

Galanin, M. D. (1995). Luminescence of Molecules and Crystals, Cambridge International Science Publishing.

Gao, L.; Zhaou, X. \& Ding, Y. (2007). Effective thermal and electrical conductivity of carbon nanotube composites. Chemical Physics Letters Vol. 434, pp. 297- 300.

Gingold, D. B. \& Lobb, C. J. (1990). Percolative conduction in three dimensions. Physical Review B, Vol. 42, pp. 8220- 8224.

Gordon, M. (1962). Good's theory of cascade processes applied to the statistics of polymer distributions. Proceeding Royal Society A, Vol. 268, pp. 240-256.

Herculus, D. M. (1965). Fluorescence and Phosphorescence Analysis, Wiley Interscience, New York

Herrman, H. J.; Derrida, B. \& Vannimenus, J. (1984). Superconductivity exponents in two and three dimensional percolation. Physical Review B, Vol. 30, pp. 4080- 4082.

Hermann, H. J. (1986). Geometrical cluster growth models and kinetic gelation. Physics Report, Vol.136, pp.153-224.

Hill D. E.; Lin, Y.; Rao, A. M.; Allard, L. F. \& Sun, Y.-P. (2002). Functionalization of Carbon nanotubes with Polystyrene, Macromolecules, Vol.35, pp.9466-9471.

Hu, G.; Zhao, C.; Zhangi, S.; Yang, M.; Wang, Z. (2006). Low percolation thresholds of electrical conductivity and rheology in poly (ethylene terephthalate) through the networks of multi- walled carbon nanotubes. Polymer, Vol. 47, pp. 480- 488.

Kaya, D.; Pekcan, Ö. \& Yilmaz, Y. (2004). Direct test of the critical exponents at the sol- gel transition. Physical Review E. Vol. 69, pp. 16117(1-10).

Kymakis, E.; Alexandou, I. \& Amaratunga, G. A. J. (2002). Single-walled carbon nanotubepolymer composites: electrical, optical and structural investigation, Synthetic Metals, Vol. 127, pp. 59- 62.

Lau, K. T. \& Hui, D. (2002). The revolutionary creation of new advanced materials- carbon nanotube composites. Composites Part B, Engineering, Vol. 33, pp. 263-277.

lijima, S. (1991). Helical microtubules of graphitic carbon. Nature, Vol. 354, pp.56.

Li, X.; Guan, W.; Yan, H. \& Huang, L. (2004). Fabrication and atomic force microscopy/ friction force microscopy studies of polyacrylamide-carbon nanotubes copolymer thin films. Materials Chemistry and Physics, Vol. 88, pp. 53- 58.

Macosko, C. W. \& Miller, D. R. (1976). A new derivation of Average Molecular Weights of Nonlinear Polymers, Macromolecules, Vol. 9, pp. 199- 206. 
Moniruzzaman, M. \& Winey, K. I. (2006). Polymer nanocomposites containing carbon nanotubes, Macromolecules, Vol. 39, pp. 5194- 5205.

Park, S. J.; Lim, S. T.; Cho, M. S.; Kim, H. M.; Joo, J. \& Choi, H.J. (2007). Electrical properties of multi-walled carbon-nanotube/poly (methyl methacrylate) nanocomposites, Current Applied Physics Vol. 5, pp. 302-304.

Pearson, D. S. \& Graessley, W. W. (1978). The structure of Rubber Networks with Multifunctional Junctions. Macromolecules, Vol. 11, pp. 528-533.

Pötschke, P., Dudkin, S. M. \& Alig, I. (2003). Dielectric spectroscopy on melt processed polycarbonate- multiwalled carbon nanotube composites. Polymer, Vol. 44, pp.50235030.

Pötschke, P.; Abdel-Goad, M.; Alig, I.; Dudkin, S. \& Lellinger, D. (2004). Rheological and dielectrical characterization of melt mixed polycarbonate-multiwalled carbon nanotube composites. Polymer, Vol. 45, pp. 8863- 8870.

Okay, O.; Kaya D. \& Pekcan, Ö. (1999). Free- radical crosslinking copolymerization of styrene and divinylbenzene: real time monitoring of the gel effect using fluorescence probe. Polymer, Vol. 40, pp. 6179- 6187.

Qian, D.; Dickey, E. C.; Andrews, R. \& Rantell, T. (2000). Load transfer and deformation mechanisms in carbon nanotube- polystyrene composites. Applied Physics Letters, Vol. 76, pp.2868-2870.

Sahimi, M. (1994). Application of Percolation Theory, Taylor and Francis, London.

Seo, M. - K., Park, S.-J. (2004). Electrical resistivity and rheological behaviors of carbon nanotubes- filled polypropylene composites. Chemical Physics Letters, Vol. 395, pp.44- 48 .

Shaffer, M. S. P. \& Windle, A. H. (1999). Fabrication and Characterization of Carbon Nanotube/ Poly (vinyl alcohol) Composites. Advanced Materials, Vol. 11, pp. 937-941.

Stauffer, D.; Coniglio, A. \& M. Adam. (1982). Gelation and Critical Phenomena. Advances Polymer Science. Vol. 44, pp.103- 158.

Stauffer, D. (1985). Introduction to Percolation Theory, Taylor and Francis, London.

Stauffer, D. \& Aharony, A. (1994). Introduction to Percolation Theory, 2nd ed. Taylor\&Francis, London.

Straley, J. P. (1977).Critical exponents of conductivity of random resistor lattices. Physical Review B, Vol. 15(12), pp. 5733- 5737.

Stockmayer, W. H. (1943). Theory of Molecular Size Distribution and Gel Formation in Branched- Chain Polymers. Journal of Chemical Physics, Vol. 11, pp. 45-54.

Thostenson, E. T.; Ren, Z. F. \& Chou, T. W. (2001). Advances in the science and technology of carbon nanotubes and their composites: a review. Composition of Science and Technology, Vol. 61, pp. 1899-1912.

Tanaka, T. (1981). Gels. Scientific American, Vol.244, pp.124- 136.

Weber, M. \& Kamal, M. R. (1997). Estimation of the Volume Resistivity of Electrically Conductive Composites. Polymer Composites, Vol. 18, pp. 711- 725.

Yilmaz, Y.; Erzan, A. \& Pekcan, Ö. (1998). Critical exponents and fractal dimension at the sol- gel phase transition via in situ fluorescence experiments. Physical Review E, Vol. 58, pp. 7487- 7491.

Yilmaz Y.; Erzan A. \& Pekcan, Ö. (2002). Slow Release percolate near glass transition. European Physical Journal E, Vol. 9, pp. 135- 141.

Yılmaz, Y.; Uysal, N.; Güney, O.; Gelir, A.; Aktaş, D. K.; Göğebakan, S.\& Öner. A. (2009). Elucidation of multiple- point interactions of pyranine fluoroprobe during the gelation. Spectrochimica Acta Part A: Molecular and Biomolecular Spectroscopy Part A, Vol. 72, pp. 332- 338. 


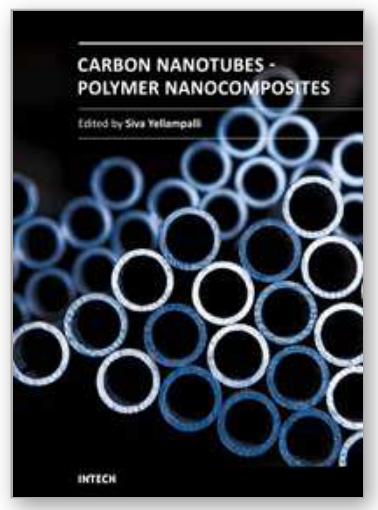

\section{Carbon Nanotubes - Polymer Nanocomposites}

Edited by Dr. Siva Yellampalli

ISBN 978-953-307-498-6

Hard cover, 396 pages

Publisher InTech

Published online 17, August, 2011

Published in print edition August, 2011

Polymer nanocomposites are a class of material with a great deal of promise for potential applications in various industries ranging from construction to aerospace. The main difference between polymeric nanocomposites and conventional composites is the filler that is being used for reinforcement. In the nanocomposites the reinforcement is on the order of nanometer that leads to a very different final macroscopic property. Due to this unique feature polymeric nanocomposites have been studied exclusively in the last decade using various nanofillers such as minerals, sheets or fibers. This books focuses on the preparation and property analysis of polymer nanocomposites with CNTs (fibers) as nano fillers. The book has been divided into three sections. The first section deals with fabrication and property analysis of new carbon nanotube structures. The second section deals with preparation and characterization of polymer composites with CNTs followed by the various applications of polymers with CNTs in the third section.

\section{How to reference}

In order to correctly reference this scholarly work, feel free to copy and paste the following:

Onder Pekcan and Gülşen Akın Evingür (2011). Conductivity Percolation of Carbon Nanotubes in Polyacrylamide Gels, Carbon Nanotubes - Polymer Nanocomposites, Dr. Siva Yellampalli (Ed.), ISBN: 978953-307-498-6, InTech, Available from: http://www.intechopen.com/books/carbon-nanotubes-polymernanocomposites/conductivity-percolation-of-carbon-nanotubes-in-polyacrylamide-gels

\section{INTECH}

open science | open minds

\section{InTech Europe}

University Campus STeP Ri

Slavka Krautzeka 83/A

51000 Rijeka, Croatia

Phone: +385 (51) 770447

Fax: +385 (51) 686166

www.intechopen.com

\section{InTech China}

Unit 405, Office Block, Hotel Equatorial Shanghai

No.65, Yan An Road (West), Shanghai, 200040, China

中国上海市延安西路65号上海国际贵都大饭店办公楼 405 单元

Phone: +86-21-62489820

Fax: $+86-21-62489821$ 
(C) 2011 The Author(s). Licensee IntechOpen. This chapter is distributed under the terms of the Creative Commons Attribution-NonCommercialShareAlike-3.0 License, which permits use, distribution and reproduction for non-commercial purposes, provided the original is properly cited and derivative works building on this content are distributed under the same license. 